\title{
Epidemiología de la Tuberculosis en el Área de Salud de Pavas, Costa Rica ${ }^{1}$
}

Giancarlo Guevara Francesa ${ }^{2}$; Monserrat Navarro Mora ${ }^{3}$; Jennyffer González Luna ${ }^{4}$

Institución: COPESALUD, Costa Rica.

\section{RESUMEN}

Este estudio tuvo por objetivo identificar los factores de riesgo asociados a la aparición de tuberculosis en Rincón Grande, Pavas. Se trata de una investigación cuantitativa realizada en dos fases: la primera correspondió a una descripción de los casos de tuberculosis en toda el área de estudio y la segunda, a un estudio de casos y controles con la población de Rincón Grande. En ambas fases se utilizó la totalidad de casos registrados de tuberculosis pulmonar entre 2010 y 2016. La fuente de datos fue el Sistema de Información Integrado de Salud y el libro de registro de casos. La selección de los controles fue aleatoria simple, con el total de sintomáticos respiratorios y el reporte del ELISA. Entre los resultados se denota que la incidencia en Pavas (periodo 2010-2016), osciló entre 10.2 y 22.2 casos por cada 100000 habitantes. El 70,4\% de los casos tenía entre 20 y 64 años de edad, el 59,0\% correspondió a hombres; el 29,6\%, a extranjeros. El 18,8\% presentó antecedentes de consumo de drogas y el $51,1 \%$ tenían estudios con escolaridad primaria o menor. Los factores asociados son hacinamiento crítico, sexo $(\mathrm{OR}=1.9, \mathrm{IC}=0.1-3.6)$, co-infección con $\mathrm{VIH}(\mathrm{OR}=9.7, \mathrm{IC}=2.3-39.6)$ y estado nutricional. Los hombres presentan 1.9 más veces la posibilidad de desarrollar tuberculosis y las personas con VIH presentan 9.7 veces dicha posibilidad. Se concluye que la seropositividad por VIH, las condiciones de hacinamiento, la desnutrición y el sexo masculino son factores de riesgo para la incidencia de tuberculosis en Rincón Grande.

Palabras clave: Epidemiología; Factores-de-riesgo; Tuberculosis.

DOI: https://doi.org/10.15517/revenf.v0i35.32174

${ }^{1}$ Fecha de recepción: 26 de enero del $2018 \quad$ Fecha de aceptación: 15 de abril del 2018
${ }^{2}$ Enfermero. Máster en Salud Pública y Epidemiología. Área de Salud de Pavas, COOPESALUD R.L. Costa Rica. Correo electrónico: gian4418@gmail.com

${ }^{3}$ Enfermera. Máster en Epidemiología. Área de Salud de Pavas, COOPESALUD R.L. Costa Rica. Correo electrónico: mmnavarro@,coopesalud.org.

${ }^{4}$ Médico. Máster en Epidemiología. Ministerio de Salud de Costa Rica, Costa Rica. Correo electrónico: jennyffer07@gmail.com 


\title{
Revista Electrónica Enfermeria Actual en costa Rica
}

\section{Epidemiology of Tuberculosis in the Pavas Health Area, Costa Rica ${ }^{1}$}

Giancarlo Guevara Francesa ${ }^{2}$; Monserrat Navarro Mora ${ }^{3}$; Jennyffer González Luna ${ }^{4}$

Institution: COPESALUD, Costa Rica.

\begin{abstract}
The objective of this study was to identify the risk factors associated with the appearance of tuberculosis in Rincón Grande, Pavas. This is a quantitative investigation carried out in two phases: the first corresponded to a description of the cases of tuberculosis in the entire study area and the second, to a case-control study with the population of Rincón Grande. In both phases, the totality of registered cases of pulmonary tuberculosis between 2010 and 2016 was used. The source of data was the Integrated Health Information System and the case record book. The selection of controls was simple random, with the total of respiratory symptoms and the ELISA report. Among the results it is noted that the incidence in Pavas (period 2010-2016), ranged between 10.2 and 22.2 cases per 100000 inhabitants. 70,4\% of the cases were between 20 and 64 years of age, 59,0\% corresponded to men; $29,6 \%$, to foreigners. $18,8 \%$ had a history of drug use and $51,1 \%$ had studies with primary or lower education. The associated factors are critical overcrowding, sex $(\mathrm{OR}=1.9, \mathrm{CI}=0.1-3.6)$, co-infection with $\mathrm{HIV}(\mathrm{OR}=9.7$, $\mathrm{CI}=2.3-39.6)$ and nutritional status. Men present 1.9 times more the possibility of developing tuberculosis and people with HIV present 9.7 times that possibility. It is concluded that HIV seropositivity, crowded conditions, malnutrition and male sex are risk factors for the incidence of tuberculosis in Rincón Grande.
\end{abstract}

Keywords: Epidemiology; Risk-factors; Tuberculosis.

DOI: https://doi.org/10.15517/revenf.v0i35.32174

${ }^{1}$ Date of receipt: January 26, 2018

${ }^{2}$ Nurse. Master's Degree in Public Health and Epidemiology. Pavas Health Area, COOPESALUD R.L. Costa Rica. E-mail: gian4418@gmail.com

${ }^{3}$ Nurse. Master's Degree in Epidemiology. Pavas Health Area, COOPESALUD R.L. Costa Rica. E-mail: mmnavarro@coopesalud.org.

${ }^{4}$ Doctor. Master's Degree in Epidemiology. Pavas Health Area. Ministry of Health of Costa Rica, Costa Rica. E-mail: jennyffer07@gmail.com 


\section{Epidemiologia da Tuberculose na Área de Saúde de Pavas, Costa Rica ${ }^{1}$}

Giancarlo Guevara Francesa ${ }^{2}$; Monserrat Navarro Mora ${ }^{3}$; Jennyffer González Luna ${ }^{4}$

Instituição: COPESALUD, Costa Rica.

\section{RESUMO}

O objetivo deste estudo foi identificar os fatores de risco associados ao aparecimento da tuberculose em Rincón Grande, Pavas. Trata-se de uma investigação quantitativa realizada em duas fases: a primeira correspondeu a uma descrição dos casos de tuberculose em toda a área de estudo e a segunda, a um estudo caso-controle com a população de Rincón Grande. Em ambas as fases, utilizou-se a totalidade dos casos registrados de tuberculose pulmonar entre 2010 e 2016. A fonte de dados foi o Sistema Integrado de Informação em Saúde e o livro de registro de casos. A seleção dos controles foi aleatória simples, com o total de sintomas respiratórios e o laudo ELISA. Entre os resultados, observa-se que a incidência em Pavas (período 2010-2016) variou entre 10,2 e 22,2 casos por 100 mil habitantes. 70,4\% dos casos tinham entre 20 e 64 anos de idade, 59,0\% correspondiam a homens; $29,6 \%$ para estrangeiros. 18,8\% tinham histórico de uso de drogas e $51,1 \%$ tinham estudos com ensino primário ou inferior. Os fatores associados são superlotação crítica, sexo $(\mathrm{OR}=1,9, \mathrm{IC}=0,1-3,6)$, co-infecção por HIV (OR $=9,7, \mathrm{IC}=2,3-39,6)$ e estado nutricional. Os homens apresentam 1,9 vezes mais a possibilidade de desenvolver tuberculose e as pessoas com HIV apresentam 9,7 vezes essa possibilidade. Conclui-se que a soropositividade ao HIV, condições de superlotação, desnutrição e sexo masculino são fatores de risco para a incidência de tuberculose em Rincón Grande.

Palavras chave: Epidemiologia; Fatores-de-risco; Tuberculose.

\footnotetext{
DOI: https://doi.org/10.15517/revenf.v0i35.32174

${ }^{1}$ Data de recepção: 26 de janeiro de 2018

Data de aceitação: 15 de abril de 2018

${ }^{2}$ Enfermeiro. Mestrado em Saúde Pública e Epidemiologia. Área de Saúde de Pavas, COOPESALUD R.L. Costa Rica. Correio eletrônico: gian4418@gmail.com

${ }^{3}$ Enfermeira. Mestrado em Epidemiologia. Área de Saúde de Pavas, COOPESALUD R.L. Costa Rica. Correio eletrônico: mmnavarro@coopesalud.org.

${ }^{4}$ Médico. Mestrado em Epidemiologia. Área de Saúde de Pavas, Costa Rica. Correio eletrônico: jennyffer07@gmail.com
} 


\section{Revista Electrónica Enfermeria Actual en costa Rica}

\section{INTRODUCCIÓN}

Desde el año 1993, la Organización Mundial de la Salud considera la tuberculosis como emergencia global, debido a que cada año aparecen nuevos casos, a lo que se suma las recaídas, abandonos del tratamiento, muerte y los contagios concomitantes en pacientes con el virus de inmunodeficiencia humana, han disparado de forma alarmante la prevalencia e incidencia de esta enfermedad, así como la mortalidad ${ }^{1}$.

Los indicadores sociodemográficos han sido identificados como factores de riesgo de contagio de esta enfermedad, entre los que sobresale la pobreza extrema, ya que favorece la desnutrición que siempre ha sido aceptada como un factor de riesgo individual ${ }^{2}$. Sin embargo, hay otros factores que influyen en la transmisión y contagio, tales como hacinamiento, migración, inadecuado control de las entidades de salud, aparición de cepas resistentes al tratamiento y género: este último llama la atención, puesto que los hombres padecen más esta enfermedad en un $70 \%$ en relación con las mujeres ${ }^{3}$. Entre otras causas que favorecen la transmisión de tuberculosis desde la perspectiva de salud, se menciona la fragmentación de los servicios y múltiples proveedores de salud, inexistencia de un plan integral de salud, diversidad en la población de pacientes, pobreza y marginalización de las poblaciones ${ }^{6}$.

La situación epidemiológica en Latinoamérica presenta un comportamiento similar a los países desarrollados, debido a la gran concentración de casos producto de la inmigración de países con alta incidencia, brotes, infectados por VIH, consumo de alcohol y drogas, condiciones de hacinamiento o pobreza extrema ${ }^{4}$. Según estimaciones de la Organización Mundial de la Salud, en el 2012, en Latinoamérica hubo 263700 nuevos casos de tuberculosis, con una tasa de incidencia de 43 por 100 000 habitantes; los casos nuevos notificados, en el mismo año, fueron 208000 con una tasa de 34 casos por 100000 habitantes que corresponde a una tasa de detección de casos del 79\%, la cual varió de manera significativa entre los países 5 .

En Costa Rica, en el periodo 2013-2014, según datos del Instituto Costarricense de Investigación y Enseñanza en Salud y Nutrición, se detectó 247 nuevos casos de tuberculosis en el 2013 y 232 en el 2014, cuya incidencia es de alrededor de ocho casos por 100 mil habitantes, lo cual sugiere un comportamiento estable en la incidencia de la enfermedad. En cuanto a su distribución, y de acuerdo con el Ministerio de Salud (2014), de las siete provincias, las que presentaron mayores tasas de incidencia (por cada 100000 habitantes) durante el 2012 fueron Limón (17.7), San José (11.9) (aquí se ubica el distrito de Pavas ${ }^{7}$ ), Puntarenas (11.8) y Guanacaste (11.5). La incidencia en el sector de Pavas, específicamente, durante el periodo comprendido entre el 2010-2016, se mantuvo entre los 10.2-22.2 casos/100.000 habitantes.

Pavas se caracteriza por tener dos sectores con marcadas diferencias socioeconómicas, Rincón Grande, al oeste, y Rohrmoser, al este. Además, el Área de Salud de Pavas (que proporciona atención a todo el distrito de Pavas) ha identificado un sector con condiciones socioeconómicas intermedias, (ubicado entre Rincón Grande y Rohrmoser). Según un estudio elaborado por la Oficina de Desarrollo Social de la Municipalidad de San José, con datos del Estado de la Nación y el Centro Centroamericano de Población, destaca que en el sector oeste del distrito, conocido como Rincón Grande se concentran las principales carencias sociales ${ }^{8}$.

El entendimiento de los patrones territoriales de la tuberculosis, así como de los factores asociados a su transmisión, permitirá caracterizar mejor el comportamiento de la enfermedad y establecer futuras estrategias de salud pública para el control de la tuberculosis en el distrito de Pavas. Es de esta forma como se planteo el objetivo de identificar los factores de riesgo asociados a la aparición de tuberculosis en Rincón Grande, Pavas. 


\section{Revista Electrónica Enfermeria Actual en costa Rica}

\section{www.revenf.ucr.ac.cr}

\section{MATERIALES Y MÉTODOS}

Según el Instituto Nacional de Estadística y Censo (2011), en todo el distrito de Pavas hay 19735 viviendas mientras que, de acuerdo con la Encuesta Nacional de Hogares (2012), se registra un total de 5861 hogares con al menos una necesidad básica insatisfecha, para una población de 19073 personas (Área de Salud de Pavas, 2015). Por otra parte, la información aportada por el personal técnico de atención primaria, para el año 2014, revela que el distrito contaba con 22225 familias distribuidas en 15962 viviendas, de las que 2291 viven en los 17 precarios del área y albergan alrededor de 18328 personas en condiciones de pobreza 9 .

Con base en el análisis de situación de salud realizado por el Área de Salud de Pavas, y con el objetivo de favorecer una atención integral y acorde con las necesidades de su población, se ha sectorizado el distrito en tres zonas conocidas como Rohrmoser, Sector intermedio (conocido como Pavas Centro) y Rincón Grande.

En cuanto a Rohrmoser, ubicado en la parte oriental del distrito, se compone principalmente de familias con rangos socioeconómicos medios y medios altos en los que el nivel educativo y el ingreso económico en general es alto, así como sus posibilidades de esparcimiento y recreación y el acceso a servicios privados de salud.

En relación con Pavas centro, su población es heterogénea, con condiciones socioeconómicas bajas y medias, habitada por un importante número de personas adultas mayores que utilizan los servicios de la seguridad social.

En lo concerniente a Rincón Grande, en 1979 inició una expansión urbana acelerada con el desarrollo de proyectos del Instituto Nacional de Vivienda y Urbanismo (INVU), del Instituto Mixto de Ayuda Social (IMAS) y de empresas privadas. Entre los proyectos más relevantes se tiene a Villa Esperanza, urbanizada en 1971; Ciudadela Libertad 1, en 1982, y Ciudadela Libertad 2, en $1984^{9}$.

En los últimos años, el área occidental del distrito ha nutrido su población a base de inmigrantes de zona rural de Costa Rica y del resto de países centroamericanos (predomina la nacionalidad nicaragüense), quienes habitan esta zona de manera permanente o temporal según sus posibilidades de trabajo, lo cual se agrava porque un porcentaje de esta población se encuentra en nuestro país en carácter de ilegalidad, lo cual dificulta su aseguramiento y adscripción formal a los servicios públicos de salud.

Con respecto a la muestra, en la primera fase, se utilizó el total de casos diagnosticados con tuberculosis pulmonar, durante todo el periodo del estudio $(n=98)$; en la segunda se aplicó lo mismo, pero en el sector específico de Rincón Grande $(n=76)$. El tamaño de la muestra del grupo control se estimó con el programa Epi info y se definió un nivel de confianza del 95\%, una potencia del 72\%, relación de caso: control de 1:3, un porcentaje de exposición de los controles de 30\% y un Odds Ratio mínimo significativo a detectar de 2.0. Según el coeficiente de concordancia de Fleiss, se define 76 casos y 238 controles para un tamaño de muestra de 314 individuos ${ }^{10}$.

La selección de los controles se realizó de forma aleatoria simple entre el total de sintomáticos respiratorios registrados y con reporte de examen de ELISA durante el periodo de estudio. Cuando los datos estaban incompletos o no registraban, se acudió al reemplazo simple. 


\section{Revista Electrónica Enfermeria Actual en costa Rica}

Para la definición de casos, controles y criterios de inclusión y exclusión se definió dos poblaciones: i) Casos: persona a quien se le diagnosticó tuberculosis. Para el diagnóstico se utilizó la tinción de Ziehl-Neelsen como método estandarizado y validado y, en los casos necesarios, se contó con la valoración y seguimiento del médico especialista en neumología o infectología y, ii) Control: persona clasificada como sintomático respiratorio durante la búsqueda pasiva y activa, a quien se le recolectó al menos dos muestra de esputo, cuyo reporte fue que "no se observaron bacilos alcohol ácido-resistentes". Este análisis bacteriológico se realizó en el laboratorio del Área de Salud de Pavas, el cual participa en un programa de control de calidad mensual y una evaluación anual por parte del INCIENSA, que es el laboratorio nacional de referencia para esta enfermedad.

Para el estudio de los controles se consideró que cumplieran con los siguientes criterios: adscripción a un EBAIS perteneciente al Área de Salud de Pavas, indicación médica de la baciloscopía y edad igual o mayor a 10 años, con reporte de examen de ELISA durante el periodo de estudio, además de contar con ficha familiar. Por otro lado, se excluyó a los pacientes, cuyo expediente presentó al menos una de las siguientes carencias: reportes biológicamente no plausibles, falta de datos, datos mal digitados o en blanco.

Este trabajo es de tipo observacional, dividido en dos fases: en la primera, se elaboró una descripción en tiempo, lugar y persona, de los pacientes diagnosticados con tuberculosis, para toda el área de salud, durante el periodo de estudio; en la segunda, debido a las particularidades del área de salud respecto a las marcadas inequidades socioeconómicas, el acceso real al sistema público de salud, a los criterios de inclusión y exclusión utilizados y dado que la tuberculosis es una enfermedad desatendida, se realizó un estudio caso-control para identificar los factores de riesgo y su magnitud, tomando como base poblacional el sector de Rincón Grande.

Como fuente de datos, se utilizó, el expediente electrónico que incluye el sistema de información integrado de salud, el libro de registro de casos de tuberculosis y la ficha familiar, a partir de los cuales se construyó una base de datos para el registro de los datos, en donde se utilizó el programa Excel 2007.

La base de datos contenía 17 variables: condición de salud (presencia/ausencia de Tuberculosis), edad, sector, sexo, escolaridad, nacionalidad, ocupación (vínculo laboral con el sector salud); peso, talla, índice de masa corporal (IMC), presencia/ausencia de consumo de drogas, presencia/ausencia de infección con VIH, presencia/ausencia de enfermedad Diabetes Mellitus, presencia/ausencia de enfermedad de enfermedades pulmonares; presencia/ausencia de hacinamiento (donde se incluye la cantidad de personas que habitan en la vivienda y número de habitaciones), así como la condición de hacinamiento.

Para la construcción de la base de datos de los casos, la mayoría de las variables que se utilizaron se recolectaron del libro de registros de casos de tuberculosis. En cuanto a la elaboración de la base de datos de controles, su construcción se realizó generando un listado de las personas sintomáticas respiratorias a quienes se les realizaron baciloscopía durante el periodo en estudio, esto a partir del expediente electrónico del área de salud.

Las variables "escolaridad", "ocupación", "presencia/ausencia de hacinamiento" se generaron a partir de los registros obtenidos en la ficha familiar, durante la visita domiciliaria que realiza el Asistente Técnico de Atención Primaria en Salud (ATAPS). 


\section{Revista Electrónica Enfermeria Actual en costa Rica}

La variable "presencia/ausencia de hacinamiento", se construyó utilizando una relación entre el número de personas que habitan en la vivienda entre el número de dormitorios que existen en la casa, conocido como índice de hacinamiento. Posteriormente se establecieron tres categorías: hasta 2.4 - sin hacinamiento; de 2.5 a 4.9 hacinamiento medio; más de 5.0 - hacinamiento crítico. ${ }^{11}$

Para la construcción de la variable IMC se consultó, en el módulo de enfermería del expediente electrónico, el record de peso y talla de los casos y controles. Los resultados se categorizaron en cuatro áreas: personas con un IMC menor a 18.5 se identificaron como bajo peso; personas con un IMC normal presentaron rangos entre 18.5 y 24.9; personas con un IMC entre 25 y 29.9 se registraron como personas con sobrepeso. Las personas con un IMC superior a 30 fueron catalogadas como obsesas.

Con respecto a las variables relacionadas con las presencia/ausencia de diabetes mellitus, VIH y enfermedades pulmonares, se consultó el módulo de medicina del expediente electrónico. La variable "presencia/ausencia de consumo de drogas", se obtuvo de la revisión de las notas médicas anotadas en el expediente digital del área de salud.

En la fase descriptiva del estudio, se aplicó estadística descriptiva que genera tasas y proporciones para las variables de tiempo, lugar y persona (cuadro I y cuadro II).

En este estudio de caso-control, se determinó los factores asociados con la tuberculosis mediante la estimación de las razones de posibilidades (OR; por su nombre en inglés Odds Ratio), utilizando para ello la regresión logística no condicional. El procedimiento logístico consistió en un análisis bivariado, seguido de uno multivariado. Las variables con un valor $\mathrm{p}=0.20$ en el análisis bivariado fueron seleccionadas para el análisis multivariado, el cual se realizó siguiendo la estrategia de paso a paso en reversa (backward stepwise) ${ }^{10}$.

Luego de probar cada modelo, se extrajo la variable con menor significancia, examinada de acuerdo con la prueba de Wald y sus intervalos de confianza ajustados y se comparan los coeficientes con el modelo anterior. Este proceso de eliminación, reajuste y verificación continúa hasta que todas las variables en el modelo son significativas o actúan como confusoras. Para el análisis estadístico se utilizó el programa Info Stat 2016.

\section{Consideraciones Éticas}

El estudio contó con la revisión y aprobación del Comité Académico Local de la Clínica de Pavas, administrada por COOPESALUD R.L. En la investigación se respetó el anonimato al mantener la confidencialidad de los datos de las personas que fueron incluidas en la selección, en el tanto en que se omitió su nombre, número de identificación u otro dato vinculado con su identidad. Para el reconocimiento de las personas como casos y controles, se estableció una codificación que mezclaba letras con numeración arábiga. 


\section{RESULTADOS}

\section{Características de los casos}

En el área de estudio, hubo un total de 112 casos de tuberculosis entre el 2010 y el 2016, de los cuales el $87.5 \%$ corresponde a tuberculosis pulmonar, con una incidencia entre 10.2 y 22.2 casos por cada 100000 habitantes (Tabla 1).

Tabla 1. Distribución de casos de tuberculosis intra y extrapulmonar (tasa por 100 mil habitantes) en el Área de Salud de Pavas, periodo 2010-2016

\begin{tabular}{c|c|c|c|c|c|c|c|c}
\hline \multirow{2}{*}{ Año } & \multicolumn{2}{|c|}{ Ambas formas } & \multicolumn{3}{|c|}{ Pulmonar } & \multicolumn{3}{c}{ Extra pulmonar } \\
& Casos & Tasa & Casos & \% & Tasa & Casos & $\%$ & Tasa \\
\hline $\mathbf{2 0 1 0}$ & 17 & 14.80 & 15 & 88,24 & 13.06 & 2 & 11,76 & 1.74 \\
$\mathbf{2 0 1 1}$ & 14 & 11.87 & 12 & 85,71 & 10.17 & 2 & 14,29 & 10 \\
$\mathbf{2 0 1 2}$ & 19 & 26.32 & 16 & 84,21 & 22.17 & 3 & 15,79 & 4.16 \\
$\mathbf{2 0 1 3}$ & 17 & 21.71 & 16 & 94,12 & 20.44 & 1 & 5,88 & 1.28 \\
$\mathbf{2 0 1 4}$ & 14 & 17.64 & 13 & 92,86 & 16.38 & 1 & 7,14 & 1.26 \\
$\mathbf{2 0 1 5}$ & 16 & 19.90 & 14 & 87,50 & 17.41 & 2 & 12,50 & 2.49 \\
$\mathbf{2 0 1 6}$ & 15 & 17.84 & 12 & 80,00 & 14.27 & 3 & 20,00 & 3.57 \\
\hline Total & 112 & 17.86 & 98 & 87,50 & 15.63 & 14 & 12,50 & 2.23 \\
\hline
\end{tabular}

Fuente: elaboración propia

De acuerdo con la tabla 1 , el 12,5\% restante corresponde a manifestaciones extrapulmonares, dentro de las que destacan afecciones ganglionares, intestinales, miliares, cutáneas, renales, meníngeas, pleurales y diseminadas. Las tasas de incidencia oscilan entre 1.3 y 4.2 casos por cada 100000 habitantes.

En la figura 1, se muestra el comportamiento de la tuberculosis pulmonar en el distrito de Pavas, en la que se observa que la mayor frecuencia de casos se presenta en los sectores con mayores situaciones de vulnerabilidad socioeconómica, al noroeste del distrito. 
Figura 1. Incidencia de casos de tuberculosis pulmonar. Área de Salud de Pavas, 2010-2016.

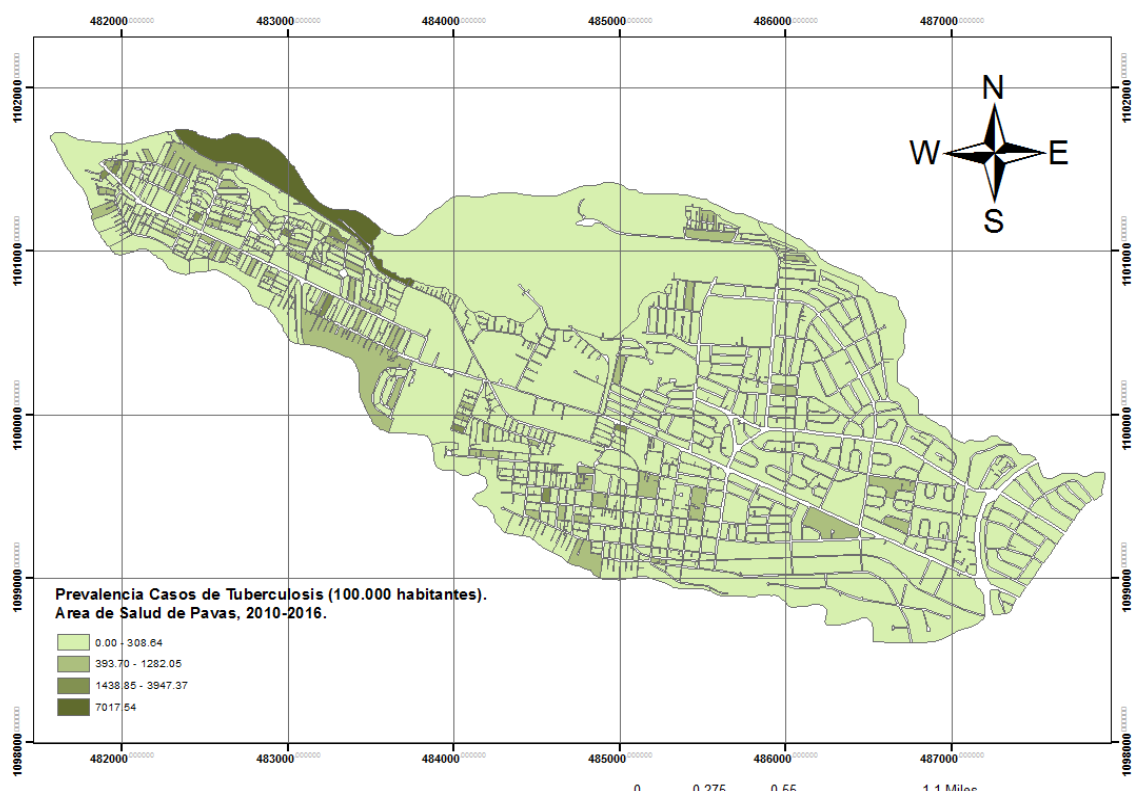

Fuente: elaboración propia.

Durante los seis años que abarca el estudio, de los 98 casos hubo una mayor presencia de la enfermedad en los hombres (59,2\%), respecto de las mujeres. En cuanto a los grupos etarios, se identificó que el grueso de los casos tenía edades entre 20 y 64 años (70,4\%), mientras que los menores de 10 fueron los menos incidentes. El 70,4\% correspondió a costarricenses. En cuanto a los extranjeros diagnosticados, en su totalidad son nicaragüenses. E1 $49 \%$ de las personas diagnosticadas presentó un índice de masa corporal (IMC) normal, mientras que el 32,6\% tuvo un IMC menor a 19, catalogado como bajo peso. En cuanto a los antecedentes personales estudiados, el 18,4\% presentó historial de consumo de drogas (consumo de alcohol, tabaco, marihuana, cocaína y/o crack); el $13 \%$ padece diabetes, el 16\% tenía enfermedades respiratorias y el $2.0 \%$ trabajaba como personal de salud. Entre el $15,3 \%$ y el $29,6 \%$ de los casos vivían en condición de hacinamiento (crítico y medio) cuando fueron diagnosticados con la enfermedad (cuadro II). 
Tabla 2. Distribución de las características demográficas y personales de los 98 casos de tuberculosis pulmonar en el Área de Salud de Pavas, periodo 2010-2016

\begin{tabular}{l|l|r|r}
\hline \multicolumn{1}{c|}{ Variable } & \multicolumn{1}{c|}{ Estrato } & \multicolumn{1}{c}{ N } \\
\hline Sexo & Masculino & 58 & 59,20 \\
Edad & Femenino & 40 & 40,80 \\
& Menor & 8 & 8,16 \\
& Adolescente & 12 & 12,25 \\
Nacionalidad & Adulto de 20 a 64 años & 69 & 70,41 \\
\multirow{5}{*}{ Escolaridad } & Adultos mayor & 9 & 9,18 \\
& Costarricense & 69 & 70,41 \\
& Extranjero & 29 & 29,59 \\
Estado nutricional & Analfabeta & 4 & 4,08 \\
& Primaria y menor & 56 & 57,14 \\
& Secundaria & 27 & 27,56 \\
Antecedentes personales & Universidad & 11 & 11,22 \\
& Bajo peso & 32 & 32,65 \\
& Normal & 48 & 48,98 \\
& Sobrepeso & 13 & 13,27 \\
& Obesidad & 5 & 5,10 \\
& Toxicómano & 18 & 18,37 \\
& Diabetes mellitus & 13 & 13,27 \\
& Patología respiratoria & 16 & 16,33 \\
& Trabajador de la salud & 2 & 2,04 \\
& Critico & 15 & 15,31 \\
& Medio & 29 & 29,59 \\
& Sin hacinamiento & 54 & 55,10 \\
\hline \multirow{5}{*}{ Condición de hacinamiento } & &
\end{tabular}

Fuente: elaboración propia

Respecto de las características de la enfermedad, el 76,5\% de los casos diagnosticados fue catalogado como sintomático respiratorios (personas con un cuadro clínico de tos con flemas con 15 días de evolución), mientras que el restante 23,5\% no contaba con tos con flemas. El 90,8\% de los casos corresponde a casos nuevos (personas que nunca han recibido tratamiento antituberculoso o lo ha recibido menos de un mes). Un $5,1 \%$ de los casos volvió a presentar la enfermedad, pese al cumplimiento completo del tratamiento antifímico.

En cuanto al método diagnóstico, el $61.2 \%$ presentó baciloscopias positivas, mientas que el $28.6 \%$ ameritó un cultivo del esputo para el establecimiento del diagnóstico, procesado por el Hospital San Juan de Dios. Del total 


\section{Revista Electrónica Enfermeria Actual en costa Rica}

de los casos, solo un 14.3\% con diagnóstico pulmonar presentó manifestaciones del Mycobacterium en otros órganos.

El porcentaje de éxito de tratamiento en el distrito es de un $71,4 \%$ que corresponde a las personas que egresaron con esputos negativos y tratamiento completo. En cuanto al tratamiento farmacológico de elección, el $66,3 \%$ fue medicado con un esquema de medicamentos acortado, que incluye dos fases de tratamiento: uno inicial con una duración de dos meses, que incluye rifampicina, isoniacida, etambutol y pirazinamida y una fase de continuación por cuatro meses que implica la ingesta de rifampicina e isoniacida. El 58,2\% inició la ingesta del tratamiento farmacológico en el Área de Salud de Pavas. De los 98 casos, se le realizó pruebas de sensibilidad a 34 muestras, de las cuales se identificó resistencia a la rifampicina $(2,0 \%)$, isoniacida $(1,0 \%)$ y estreptomicina $(1,0 \%)$. Finalmente, de los 98 casos, a 96 se les aplicó el examen de ELISA (Enzyme Linked Immunosorbent Assay), con un $12,2 \%$ de casos positivos con VIH.

Respecto de los factores de riesgo se observa en la tabla 3, las variables de sexo, nacionalidad, VIH, estado nutricional, toxicomanía y hacinamiento en Rincón Grande fueron asociadas, de manera independiente y aislada, con la tuberculosis, con valores de significancia menores a 0.2 , por lo que fueron incluidas en el modelo multivariado de la tabla 4.

A partir del modelo se estableció que las personas con tuberculosis pulmonar tuvieron 9.7 veces la posibilidad de tener la condición de ser VIH positivos (IC 95\%: 2.4-39.6).

Con respecto al estado nutricional, se identificó que las personas que presentan sobrepeso tienen 0.02 veces menos posibilidades de presentar tuberculosis en relación con las personas con bajo peso (IC 95\%: 0.0-0.1). Por otro lado, las personas que no viven en hacinamiento tienen 0.3 veces menos posibilidades de desarrollar un diagnóstico de tuberculosis si se les compara con quienes sí viven en esa condición. (IC 95\%: 0.1-0.9).

En cuanto al sexo, se identificó que los hombres presentan 1.9 veces más posibilidades de tener tuberculosis en comparación con las mujeres (IC 95\%: 0.1-3.6).

Dada la naturaleza de la cobertura de la clínica y los criterios de inclusión y exclusión, a pesar que la muestra refleja muy bien la población atendida por el área de salud de Rincón Grande, podría haber un sesgo de selección por edad, por lo cual se excluyó del modelo de análisis. El resto de variables se excluye dado que no se asocian epidemiológicamente sobre un valor de decisión de significancia de 0.1 (Tabla 4). 
Tabla 3. Distribución de los factores de riesgo para tuberculosis pulmonar, mediante modelos no ajustados, en el Rincón Grande de Pavas, periodo 2010-2016

\begin{tabular}{|c|c|c|c|c|c|}
\hline Variable & Casos & Controles & OR & $95 \%$ IC & Valor-p \\
\hline \multicolumn{6}{|l|}{ Sexo } \\
\hline Femenino & 32 & 155 & & & \\
\hline Masculino & 44 & 83 & 2.57 & $1.51-4.35$ & 0.0005 \\
\hline \multicolumn{6}{|l|}{ Nacionalidad } \\
\hline Extranjero & 26 & 50 & & & \\
\hline Nacional & 50 & 188 & 0.51 & $0.29-0.90$ & 0.0205 \\
\hline \multicolumn{6}{|l|}{ Escolaridad } \\
\hline Analfabeta & 4 & 7 & & & \\
\hline Primaria y menor & 45 & 125 & 0.63 & $0.18-2.25$ & 0.4775 \\
\hline Secundaria & 22 & 95 & 0.41 & $0.11-1.51$ & 0.1776 \\
\hline Universidad & 5 & 11 & 0.80 & $0.16-4.02$ & 0.7820 \\
\hline \multicolumn{6}{|l|}{ Estado nutricional } \\
\hline Bajo Peso & 24 & 4 & & & \\
\hline Normal & 36 & 70 & 0.09 & $0.03-0.27$ & $<0.0001$ \\
\hline Obesidad & 4 & 78 & 0.01 & $0.00-0.04$ & $<0.0001$ \\
\hline Sobrepeso & 12 & 86 & 0.02 & $0.01-0.08$ & $<0.0001$ \\
\hline \multicolumn{6}{|c|}{ Condición de hacinamiento } \\
\hline Crítico & 12 & 13 & & & \\
\hline Medio & 26 & 65 & 0.43 & $0.17-1.07$ & 0.0707 \\
\hline Sin hacinamiento & 38 & 160 & 0.26 & $0.11-0.61$ & 0.0020 \\
\hline \multicolumn{6}{|l|}{ VIH } \\
\hline Negativo & 65 & 234 & & & \\
\hline Positivo & 9 & 4 & 8.10 & $2.42-27.15$ & 0.0007 \\
\hline \multicolumn{6}{|l|}{ Toxicómano } \\
\hline No & 63 & 218 & & & \\
\hline Sí & 13 & 20 & 2.25 & $1.06-4.77$ & 0.0347 \\
\hline \multicolumn{6}{|l|}{ Diabetes mellitus } \\
\hline No & 66 & 218 & & & \\
\hline Sí & 10 & 20 & 1.65 & $0.74-3.70$ & 0.2233 \\
\hline \multicolumn{6}{|l|}{ Patología respiratoria } \\
\hline No & 64 & 192 & & & \\
\hline Sí & 12 & 46 & 0.78 & $0.39-1.57$ & 0.4897 \\
\hline
\end{tabular}

Fuente: elaboración propia 
Tabla 4. Distribución de los factores de riesgo para tuberculosis pulmonar, en el Rincón Grande de Pavas, período 2010-2016: estudio de caso-control

\begin{tabular}{|c|c|c|c|}
\hline Variable & OR & $95 \%$ IC & Valor-p \\
\hline \multicolumn{4}{|l|}{ VIH } \\
\hline \multicolumn{4}{|l|}{ Negativo } \\
\hline Positivo & 9.73 & $2.39-39.57$ & 0.0015 \\
\hline \multicolumn{4}{|l|}{ Estado nutricional } \\
\hline \multicolumn{4}{|l|}{ Bajo Peso } \\
\hline Normal & 0.07 & $0.02-0.23$ & $<0.0001$ \\
\hline Obesidad & 0.01 & $0.00-0.04$ & $<0.0001$ \\
\hline Sobrepeso & 0.02 & $0.01-0.09$ & $<0.0001$ \\
\hline \multicolumn{4}{|c|}{ Condición de hacinamiento } \\
\hline \multicolumn{4}{|l|}{ Crítico } \\
\hline Medio & 0.45 & $0.15-1.37$ & 0.1596 \\
\hline Sin hacinamiento & 0.31 & $0.11-0.88$ & 0.0286 \\
\hline \multicolumn{4}{|l|}{ Sexo } \\
\hline Femenino & & & \\
\hline Masculino & 1.87 & $0.98-3.56$ & 0.0587 \\
\hline
\end{tabular}

Fuente: elaboración propia

\section{DISCUSIÓN}

En el presente estudio se investigó el comportamiento de la tuberculosis en el distrito de Pavas y los factores asociados en el área de Rincón Grande, sector que incluye situaciones de pobreza, dificultad para acceder a sistema de salud, educación o trabajo, lo que podría suponer una relación paralela de la enfermedad con respecto al lugar de aparición de los casos.

En el periodo de estudio, se presentó una incidencia de hasta 22 casos por cada 100000 que sobrepasa, en gran medida, el registro a nivel nacional desde el que se afirma que, para el 2015, la tasa de incidencia se mantuvo en 11 personas con tuberculosis por cada 100000 habitantes $^{12}$.

En cuanto a la incidencia acumulada por sectores, se observa que existen zonas dentro del distrito en donde se presenta un mayor número de casos de la enfermedad. En la representación gráfica de Pavas, se identifica un aumento considerable de la prevalencia al noroeste del distrito, que podría relacionarse con niveles de pobreza, hacimiento, dificultades para el acceso a la alimentación, seguridad social, educación y trabajos profesionales, los cuales aumentan hacia ese sector y que coincide con lo que plantea la OPS/OMS, al afirmar que la tuberculosis es un problema asociado a la pobreza y las malas condiciones de vida, sumado a las dificultades de acceso a los servicios de salud, por lo que requiere ser abordado por toda la sociedad ${ }^{12}$. En este caso, tras analizar las 


\section{Revista Electrónica Enfermeria Actual en costa Rica}

condiciones de vida de los habitantes de Pavas, se identificó varios grupos sociales, económicos y culturales con grandes heterogeneidades poblacionales: tales determinantes sirven como factores protectores o de riesgo para el desarrollo de la enfermedad.

Para poder entender teóricamente la influencia de los factores protectores y de riesgo, fue necesario utilizar herramientas asociadas a la epidemiología social, por ende, se hizo una revisión del tema de determinantes sociales de la salud, definidos por la Organización Mundial de la Salud como "un conjunto de factores personales, sociales, económicos y ambientales que determinan el estado de salud de los individuos o poblaciones" ${ }^{13}$. Dado lo anterior, es una necesidad que los profesionales analicen y generen estrategias de intervención a partir de los comportamientos y los estilos de vida saludables, los ingresos y la posición social, la educación, el trabajo y las condiciones laborales, el acceso a servicios sanitarios adecuados y los entornos físicos. Al visualizar las relaciones que generan los diferentes determinantes entre sí, se entiende mejor las condiciones de vida que ejercen un evidente impacto sobre la salud: los cambios en estos estilos de vida y condiciones de vida, que determinan el estado de salud, se consideran resultados intermedios de salud ${ }^{13}$.

A partir del análisis de los determinantes sociales de la salud se puede explicar la multicausalidad en el desarrollo de la enfermedad de la tuberculosis y la razón por la que tiene una aparición diversa en el distrito.

La mayoría de los determinantes (a excepción del consumo de drogas y el tema de las comorbilidades) corresponden a determinantes estructurales de la salud o determinantes sociales de inequidad de salud, según lo expone el modelo de la Organización Mundial de la Salud, los cuales son aquellos que generan la estratificación social, a partir de la que se condicionan oportunidades de salud de grupos sociales tomando en cuenta las jerarquías de poder, prestigio y acceso a los recursos: algunos de ellos son el ingreso, la educación, el género, etnicidad y sexualidad ${ }^{13}$.

En cuanto al caso en estudio, el distrito presenta diferencias importantes en sus grupos poblacionales, entre las que la principal sería la situación económica, respecto de lo que cabe mencionar que las condiciones de pobreza extrema en Rincón Grande se vinculan con las dificultades para tener viviendas adecuadas y sin condiciones de hacinamiento, así como una alimentación variada y balanceada que permita tener un buen estado nutricional y contar con posibilidades de acceder a la seguridad pública o a servicios de salud privados u oportunidades de superación académica, a las que se suma características como el sexo, la edad o la nacionalidad, imposibles de modificar pero que impactan, positiva o negativamente, el desarrollo de la enfermedad.

El modelo de determinantes establece que el papel del sistema de salud se hace relevante a través del problema del acceso e incorpora diferencias en la exposición y la vulnerabilidad. En el tema de la tuberculosis, el sistema de salud le proporciona cobertura al $100 \%$ a la población diagnosticada con la enfermedad, así como el seguimiento y el tratamiento, lo cual se constata en la estadística descriptiva. 


\section{Revista Electrónica Enfermeria Actual en costa Rica}

El sistema de salud puede orientarse directamente a las diferencias en la exposición y la vulnerabilidad, no solo mejorando el acceso equitativo a la atención, sino promocionando la acción intersectorial para mejorar el estado de salud, lo que se visibiliza en el esfuerzo del sistema en cuanto a articular con otras instituciones y niveles de atención.

En lo que respecta a la relación entre sexo y tuberculosis, para determinar que esa condición es un factor de riesgo para la tuberculosis, en la investigación de Godoy et al. se asoció el género masculino (OR 1.8; IC del 95\%, 1.32.6) con una incidencia superior, comportamiento que se constata en otros estudios y que se considera un factor que continúa presentándose pese a que se neutraliza la influencia de otros factores como la edad, la infección por el VIH, dependencia a drogas IV y el antecedente de ser privado de libertad ${ }^{14}$. Por otra parte, se ha demostrado una mayor transmisión de la infección de la tuberculosis en los contactos masculinos de casos BK positivos ${ }^{15}$.

Luego, en el año 2007, un estudio en Cuba desarrollado identificó como factor de riesgo el hacinamiento que presentó una relación causal importante $(\mathrm{OR} 10,1 \mathrm{p}=0,0001)$ y que concuerda con los resultados del presente estudio $^{16}$.

Respecto del hacinamiento beneficia la transmisión de M. tuberculosis, puesto que la distancia entre el caso como fuente emisora de bacilos y el contacto incide en la posibilidad de contagio -lo cual se corrobora en diferentes estudios ${ }^{17}$ - aunque el riesgo de transmisión disminuye de forma logarítmica a partir del medio metro.

Por otro lado, se ha determinado que la exposición al factor del estado nutricional con IMC $\leq 19,9$ presenta 19 veces más probabilidades de padecer la enfermedad en comparación con los no expuestos, por ende, quedó demostrada la asociación de causalidad con el cálculo del intervalo de confianza (2,51-186.51) y el valor de la prueba de X2 obtenido $(\mathrm{p}=0,01)$, puesto que la nutrición apropiada resulta indispensable para el buen funcionamiento del sistema inmunológico ${ }^{18}$.

Hay un vínculo ineludible entre desnutrición y disminuir la resistencia a la infección, ya que se ha comprobado reiteradamente que el grado de compromiso inmunológico depende del grado de malnutrición caloricoproteica, de la presencia de infección y de la edad de la instauración de la desnutrición, la cual deprime la producción de anticuerpos, la función de las células fagocíticas, los niveles de complemento y la respuesta mediada por linfocitos T, condición asociada con un aumento de la susceptibilidad a las infecciones por microorganismos ${ }^{19}$.

Finalmente, en cuanto a la asociación de la tuberculosis y el VIH, hay consistencia entre los resultados de este estudio y aquellos que respaldan que personas con VIH tenían 29 veces más la posibilidad de enfermarse de tuberculosis (22.7-37.0 $)^{20}$. Se ha identificado que la infección por el virus de inmunodeficiencia humana (VIH) se constituye en el principal factor de riesgo para desarrollar tuberculosis activa, mientras que la tuberculosis es el principal marcador de pronóstico de la infección por VIH en los países en desarrollo ${ }^{21}$. Al respecto, la OMS declara que, al menos un tercio de los 38,6 millones de personas que hay en el mundo infectadas por el VIH también están infectadas por el bacilo de la tuberculosis, las cuales corren un riesgo mucho mayor de padecer 


\section{Revista Electrónica Enfermeria Actual en costa Rica}

tuberculosis activa, ya que el VIH debilita el sistema inmunitario y ello aumenta la probabilidad de que la infección latente por tuberculosis pase a enfermedad activa, máxime considerando que los pacientes infectados por VIH tienen una probabilidad de hasta 50 veces mayor de sufrir tuberculosis a lo largo de su vida, en comparación con los no infectados ${ }^{12}$.

Es necesario mencionar que dada la naturaleza de la cobertura de la clínica, y los criterios de inclusión y exclusión y a pesar de la que la muestra refleja muy bien a la población atendida por el Área de Salud en el sector de Rincón Grande, podría haber un sesgo de selección por edad, por ende, se excluyó del modelo de análisis, además de que se excluye del análisis multivariable las que no se asocien epidemiológicamente sobre un valor de decisión de significancia de 0.1 .

\section{CONCLUSIONES}

El distrito de Pavas cuenta con importantes determinantes, respaldados teóricamente, que aumentan la prevalencia de la TB en el sector, especialmente en Rincón Grande.

Se pudo identificar que la seropositividad por VIH, vivir en condiciones de hacinamiento, mantener un estado de desnutrición y el sexo masculino son factores de riesgo asociados a la incidencia de tuberculosis en el sector de Rincón Grande. Esta situación refleja una transmisión activa de la enfermedad y además la complejidad a la cual se enfrenta el sistema de salud cuando atiende esta patología, por lo que se recomienda acciones específicas destinadas a la prevención del VIH, las cuales se enlacen el área de salud, con ONG y grupos o redes organizadas de atención de VIH para destinar sus proyectos a trabajar el tema en Rincón Grande.

La información epidemiológica recabada es de suma importancia para las personas tomadoras de decisiones para definir e implementar acciones dentro de un plan de trabajo dirigido a solventar necesidades y, por ende, disminuir las posibilidades de aparición de casos nuevos de tuberculosis en este sector en particular.

El estado nutricional en una población está en definitiva influenciado por su grado de educación al respecto y la capacidad adquisitiva con que se cuente, por lo que se considera se debe reforzar con una consulta nutricional a todos aquellos pacientes que se encuentren en riesgo de padecer TB.

En factores como el hacinamiento existe una relación directa con las condiciones socioeconómicas de la población en estudio, y de igual forma es responsabilidad del sistema de salud re-direccionar o crear acciones destinadas a mejorar las condiciones de pobreza o pobreza extrema que se encuentran en estos sectores de la población. 


\section{Revista Electrónica Enfermeria Actual en costa Rica}

La incidencia de Pavas -que duplica la nacional- precisa un análisis sobre la posibilidad de realizar un estudio adicional que tome en cuenta un posible ajuste o estandarización de la población, de manera que se facilite la comparación de las medidas resumen entre el distrito y otros sectores con características similares.

\section{Declaración de conflicto de intereses}

Los autores declaran que no tienen conflictos de intereses personales, económicos, laborales ni de ninguna otra índole.

\section{REFERENCIAS BIBLIOGRÁFICAS}

1. Chamizo H, Salas P. Estudio del contexto de riesgo de la tuberculosis: una perspectiva ecológica. Revistas de Ciencias Administrativas y Financieras de la Seguridad Social. 2005; 13: 83-98.

2. Molina SI, Cándido LP, Hernández R. Un estudio ecológico sobre tuberculosis en un municipio de Cuba. Revista de Salud Pública. 2003; 19 (5):1305-1312.

3. Lancet. Tackling poverty in tuberculosis control. Revista Lancet. 2005; 366: 9503-2063.

4. Organización Mundial de la Salud. Ethics guidance for the implementation of the end tb strategy. Ginebra. Editorial Organización Mundial de la Salud. 2017; 1: 10.

5. Organización Mundial de la Salud. Marco de trabajo para el control de la tuberculosis en las ciudades de Latinoamérica y del Caribe. Washington DC. Editorial Organización Mundial de la Salud. 2014; 1: 7.

6. Pérez A. Eliminación de la Tuberculosis como problema de salud pública, una elección acertada. Revista Española de Salud Pública. 2007; 81: 59-62.

7. Ministerio de Salud de Costa Rica. Análisis de Situación de Salud en Costa Rica. Editorial Ministerio de Salud de Costa Rica. 2014; 1: 83-84.

8. Chacón L. El precarismo: análisis histórico y su desarrollo en el distrito de Pavas, San José, Costa Rica. Revista Costarricense de Ciencias Médicas. 1999; 20: 195-213.

9. Área de Salud de Pavas. Análisis de la Situación Integral de Salud. San José. s.e. San José. 2015; 36-37, 45-48, 67-68.

10. Fleiss J. Statistical Methods for Rates and Proportions. Ed. 3, New York. Editorial Wiley. 1981; 1: 660.

11. Muñoz C. "Requerimientos de vivienda: Comparación metodologías utilizadas por Mideplan. Ministerio de Planificación de Chile. 2008; 11: 1-29. 


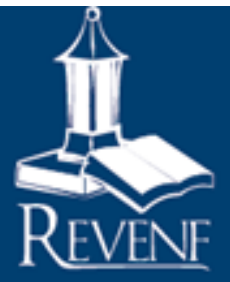

\section{Revista Electrónica Enfermeria Actual en costa Rica}

12. Organización Mundial de la Salud. Perfil de tuberculosis de Costa Rica. Organización Mundial de la Salud, 2007.

13. Moiso A. Determinantes de la Salud. En H. Barragán, Fundamentos de Salud Pública. Editorial de la Universidad Nacional de La Plata. 2007; 1:160-189.

14. Godoy P, Nogues A, Alsedà M, Manonelles A, Artigues A, García M. Factores de riesgo asociados a pacientes tuberculosis con microscopía del esputo positiva. Gaceta Sanitaria. 2001; 15: 506-512.

15. Vidal R, Miravitlles M, Caylá J, Torrella M, Martín N, De Gracia J. Estudio del contagio en 3.071 contactos familiares de enfermos con tubeculosis. Medicina clínica. 1997; 108: 361-365.

16. Machado PH, Valdés S, González E, García E. Riesgo de enfermar de tuberculosis de los convivientes adultos de enfermos bacilíferos. Revista Cubana de Medicina Tropical. 2007; 59.

17. Bayarri M, San Martín F. Tuberculosis pulmonar como enfermedad profesional. Archivos de Bronconeumología. 2004; 40: 463-472.

18. Serpa I, Pardo C. Hernández R. Un estudio ecológico sobre tuberculosis en un municipio de Cuba. Cad. Saúde Pública. 2003; 19: 1305-1312.

19. Lozano L, Plasencia C, Ramos D, García R, Mahíquez LO. Factores de riesgo socioeconómicos de la tuberculosis pulmonar en el municipio de Santiago de Cuba. Medisan. 2009. 13 (4): 1-6.

20. Ayala G. Inmunidad y nutrición: inmunonutrientes. Farmacia profesional. 2006; 20: 2-57.

21. García C. Tuberculosis en grupos de riesgo en la Región Metropolitana: 2008. Revista chilena de enfermedades respiratorias. 2010; 26: 105-111.

22. Corbett EL, Watt CJ, Walker N, Maher D, Williams BG, Raviglione MC, Dye C. The growing burden of tuberculosis: global trends and interactions with the HIV epidemic. Archives of internal medicine. 2003; 163: 1009-1021. 